\title{
Análise do estudo anatomopatológico de pacientes submetidos a cirurgia valvar no InCór - HC FMUSP
}

\author{
Hélio A. FABRI*, Pablo M. A. POMERANTZEFF*, Ana Cristina M. MONTEIRO*, Paulo GUTIERREZ*, \\ Raimunda VIOLANTE*, José Otávio C. AULER JÚNIOR*, Max GRINBERG*, Geraldo VERGINELLI*, \\ Adib D. JATENE*
}

RBCCV 44205-185

\begin{abstract}
FABRI, H. A.; POMERANTZEFF, P. M. A.; MONTEIRO, A. C. M.; GUTIERREZ, P.; VIOLANTE, R.; AULER JÚNIOR, J. O. C.; GRINBERG, M.; VERGINELLI, G.; JA TENE, A. D. - Análise do estudo anatomopatológico de pacientes submetidos a cirurgia valvar no InCór - HCFMUSP. Rev. Bras. Cir. Cardiovasc., 7(4):243$249,1992$.

RESUMO: Das 44 necropsias realizadas em pacientes submetidos a cirurgia valvar no ano de 1990 no InCór, foram encontradas como causas clínicas de óbito nos pacientes mitrais: coagulopatia e sangramento em 11 casos; baixo débito em 7 ; choque cardiogênico em 5 ; choque séptico em 3 ; falência de múltiplos órgãos em 3; síndrome da angústia respiratória do adulto (S.A.R.A.) em 2; arritmia ventricular severa em 2; tromboembolismo pulmonar (T.E.P.) em 1. Nos pacientes aórticos: coagulopatia e sangramento em 5 ; choque cardiogênico em 2; septicemia em 3; aneurisma micótico roto em 1; acidente vascular cerebral hemorrágico (A.V.C.H.) em 1; arritmia venticular em 1. Nos pacientes mitro-aórticos: coagulopatia e sangramento em 2; T.E.P. em 2; septicemia em 1; arritmia ventricular severa em 1; S.A.R.A. em 1. Encontramos como causas anatomopatológicas de óbito nos pacientes mitrais: choque hemorrágico em 13; falência de múltiplos órgảos em 4; choque séptico em 3; broncopneumonia (B.C.P.) em 4; infarto agudo do miocárdio (I.A.M.) em 3; choque cardiogênico em 3; hemorragia cerebral em 1; S.A.R.A. em 1. Nos pacientes aórticos: endocardite em 3; choque hemorrágico em 2; hemorragia cerebral em 2; I.A.M. em 1; choque cardiogênico em 1; septicemia em 1; S.A.R.A. em 1; dissecção aguda de aorta com coagulopatia em 1. Nos pacientes mitro-aórticos: falência de múltiplos órgãos em 2; T.E.P. em 2; S.A.R.A. em 1; choque hemorrágico em 1; endocardite em 1. Concluímos haver correlaçăo entre diagnóstico clínico e anatomopatológico em 36 pacientes, correspondendo a $82 \%$ dos casos, sendo a principal causa de óbito o choque hemorrágico. Uma observação importante: 11 (25\%) pacientes apresentavam alguma forma de hepatopatia crônica, nāo diagnosticada anteriormente pelo estudo clínico-laboratorial, e que alguns pacientes que evoluíram em baixo débito, nāo explicado clinicamente, tiveram como achado anatomopatológico infarto subendocárdico.
\end{abstract}

DESCRITORES: valvas cardíacas, estudo anatomopatológico.

\section{INTRODUÇĀO}

Coagulopatia, sangramento, choque cardiogênico, baixo débito, infecçāo e outros sāo situaçōes que o cirurgião cardiovascular vivencia rotineiramente e que potencialmente podem levar o paciente a óbito, mesmo com diagnóstico e tratamento precoce.
Nosso estudo mostra a relação entre as complicaçōes encontradas durante o pós-operatório em pacientes submetidos a cirurgia valvar correlacionando com os achados anatomopatológicos em 44 pacientes.

Em nosso Serviço a necropsia é feita rotineiramente. De janeiro a dezembro de 1990 foram sub-

Trabalho realizado no Instituto do Coraçáo do Hospital das Clínicas da Faculdade de Medicina da Universidade de São Paulo. São Paulo, SP, Brasil. Apresentado ao $19^{\circ}$ Congresso Nacional de Cirurgia Cardíaca. Sâo Paulo, SP, 7 a 9 de maio, 1992.

* Do Instituto do Coração do Hospital das Clínicas da Faculdade de Medicina da Universidade de São Paulo

Endereço para separatas: Hélio A. Fabri. Av. Dr. Enéas Carvalho de Aguiar, 44, Divisão Cirúrgica. 05403 São Paulo, SP, Brasil. 
FABRI, H. A.; POMERANTZEFF, P. M. A.; MONTEIRO, A. C. M.; GUTIERREZ, P.; VIOLANTE, R.; AULER JÚNIOR, J. O. C.; GRINBERG, M.; VERGINELLI, G.; JATENE, A. D. - Análise do estudo anatomopatológico de pacientes submetidos a cirurgia valvar no InCór - HC FMUSP. Rev. Bras. Cir. Cardiovasc., 7(4):243-249, 1992.

metidos a cirurgia valvar 364 pacientes com idade média de 44,76 anos, sendo operados em caráter de urgência $32(23,13 \%)$. A mortalidade global foi de $12,08 \%$, correspondendo a 44 pacientes. A mortalidade dos pacientes operados em caráter de urgência foi de $25 \%$ e a mortalidade nos casos eletivos foi de $10,84 \%$. A mortalidade dos pacientes submetidos a cirurgia valvar por endocardite infecciosa foi de $23,07 \%(6 / 26)$.

O nosso objetivo foi analisar detalhadamente os achados anatomopatológicos para determinar a correlaçāo entre este e a causa clínica do óbito.

\section{CASUÍSTICA E MÉTODOS}

Foram analisados os resultados das necropsias de 44 pacientes submetidos a cirurgia valvar no ano de 1990 correspondentes a $12,08 \%$ dos pacientes valvares operados. A idade variou de 4 a 78 anos, com média de $52,43 \%$; sendo $25(56,81 \%)$ do sexo feminino e $19(43,18 \%)$ do sexo masculino.

O diagnóstico pré-operatório foi: endocardite bacteriana em $7(15,89 \%)$ pacientes, sendo $4 \mathrm{em}$ próteses e 3 em valva natural; estenose mitral em $2(4,54 \%)$; dupla disfunção mitral 5 (11,36\%); Insuficiência mitral em $4(9,08 \%)$; estenose mitral em 3 $(6,81 \%)$; insuficiência aórtica $1(2,27 \%)$; dupla lesão aórtica 2 (4,5\%); mitro-aórticos 3 (6,81\%); mitrotricuspídeos $1(2,27 \%)$; dsfunção de uma prótese $14(31,8 \%)$; disfunçāo de prótese aórtica e mitral 1 $(2,27 \%)$; estenose aórtica associada a dissecçāo de aorta tipo I 1 (2,27\%).

Destes pacientes, $28(63,6 \%)$ foram submetidos a tratamento cirúrgico da valva mitral, sendo uma recomissurotomia mitral, 19 retrocas e 8 trocas; 11 $(25 \%)$ foram submetidos a tratamento cirúrgico da valva aórtica, sendo 7 primeira substituiçāo, 3 retroca e um paciente submetido a comissurotomia aórtica associada a ressecçāo de estenose subaórtica. Cinco $(1,36 \%)$ pacientes foram submetidos a substituiçāo das valvas mitral e aórtica, sendo 1 caso de primeira operaçāo e 4 retrocas mitro-aórticas.

Trinta e quatro por cento dos pacientes tiveram procedimentos associados (inclusive tricúspide).

Com relaçāo às reoperaçōes mitrais: 4 pacientes já haviam sido submetidos a comissurotomia, sendo, portanto, a segunda operação; 3 pacientes foram submetidos a terceira troca mitral; 4 pacientes foram submetidos a quarta troca mitral; 4 pacientes a segunda troca mitral e 2 pacientes a quarta troca mitral, sendo que a primeira operaçăo foi comissurotomia. Associado a revascularizaçāo do miocárdio, tivemos um paciente com a terceira troca de valva mitral. Nos mitro-tricuspídeos: 1 paciente submetido a retroca mitral associada a plástica de

TABELA

PRINCIPAIS CAUSAS CLÍNICAS E ANATOMOPATOLÓGICAS DE ÓBITO.

\begin{tabular}{|c|c|c|c|c|}
\hline \multicolumn{3}{|c|}{ Principais causas clínicăs de óbito } & \multicolumn{2}{|l|}{ Principais causas anatomopatológicas de óbito } \\
\hline 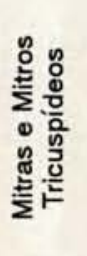 & $\begin{array}{l}\text { Coagulopatia e sangramento } \\
\text { Baixo débito } \\
\text { Choque cardiogênico } \\
\text { Choque séptico } \\
\text { Falência de múltiplos órgāos } \\
\text { S.A.R.A. } \\
\text { Arritmia ventricular severa } \\
\text { Tromboembolismo pulmonar }\end{array}$ & $\begin{array}{r}11 \\
7 \\
5 \\
3 \\
3 \\
2 \\
2 \\
1\end{array}$ & $\begin{array}{l}\text { Choque hemorrágico } \\
\text { Falência de múltiplos órgãos } \\
\text { B.C.P. } \\
\text { Choque cardiogênico } \\
\text { Choque séptico } \\
\text { Infarto do miocárdio } \\
\text { S.A.R.A } \\
\text { Hemorragia cerebral }\end{array}$ & $\begin{array}{r}13 \\
4 \\
4 \\
3 \\
3 \\
3 \\
1 \\
1\end{array}$ \\
\hline 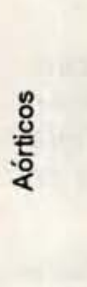 & $\begin{array}{l}\text { Coagulopatia e sangramento } \\
\text { Septicemia } \\
\text { Choque cardiogênico } \\
\text { Aneurisma cerebral micótico roto } \\
\text { A.V.C.H. } \\
\text { Arritmia ventricular severa }\end{array}$ & $\begin{array}{l}5 \\
3 \\
2 \\
1 \\
1 \\
1\end{array}$ & $\begin{array}{l}\text { Endocardite } \\
\text { Choque hemorrágico } \\
\text { Hemorragia cerebral } \\
\text { Choque cardiogênico } \\
\text { Septicemia } \\
\text { I.A.M. } \\
\text { S.A.R.A. } \\
\text { B.C.P. } \\
\text { Dissecçāo aguda de aorta com sangramento }\end{array}$ & $\begin{array}{l}3 \\
2 \\
2 \\
1 \\
1 \\
1 \\
1 \\
1 \\
1\end{array}$ \\
\hline 올 & $\begin{array}{l}\text { Coagulopatia e sangramento } \\
\text { Tromboembolismo pulmonar } \\
\text { Septicemia } \\
\text { S.A.R.A. } \\
\text { Arritmía ventricular severa }\end{array}$ & $\begin{array}{l}2 \\
1 \\
1 \\
1 \\
1\end{array}$ & $\begin{array}{l}\text { Falência de múltiplos órgāos } \\
\text { Tromboembolismo pulmonar } \\
\text { Choque hemorrágico } \\
\text { S.A.R.A. } \\
\text { Endocardite }\end{array}$ & $\begin{array}{l}2 \\
2 \\
1 \\
1 \\
1\end{array}$ \\
\hline
\end{tabular}


FABRI, H. A.; POMERANTZEFF, P. M. A.; MONTEIRO, A. C. M.; GUTIERREZ, P.; VIOLANTE, R.; AULER JÚNIOR, J. O. C.; GRINBERG, M.; VERGINELLI, G.; JATENE, A. D. - Análise do estudo anatomopatológico de pacientes submetidos a cirurgia valvar no InCór - HC FMUSP. Rev. Bras. Cir. Cardiovasc., 7(4):243-249, 1992.

De Vega na tricúspide; 1 paciente a terceira troca de valva mitral mais plástica de De Vega na tricúspide, sendo que este tinha duas comissurotomias mitrais prévias e 1 paciente a quarta troca de valva mais plástica de De Vega na tricúspide.

Nos aórticos: 1 paciente troca de valva aórtica ( $2^{\mathrm{a}}$ operaçăo); 1 paciente com troca de valva aórtica ( $3^{\text {a }}$ operaçāo) e 1 paciente com troca de valva aórtica (4ª operação). Nos mitro-aórticos: quatro trocas de valvas aórtica e mitral ( $2^{\mathrm{a}}$ operaçāo).

Tivemos, portanto, um total de $28(66,63 \%)$ reoperaçōes.

Com relação à classe funcional (New York Heart Association - NYHA) $44,72 \%$ dos pacientes encontravam-se em C.F.IV, $38,63 \%$ em C.F.III e $32,63 \%$ em C.F.II.

\section{RESULTADOS}

Considerando a ocorrência dos óbitos antes e depois de 48 horas, tivemos $12(27,27 \%)$ óbitos nas primeiras 48 horas e $24(54,54 \%)$ óbitos depois de 48 horas, além de $8(18,18 \%)$ óbitos na sala de operaçōes.

Como principais complicaçōes na sala operatória, tivemos: rotura do sulco atrioventricular (1 caso); rotura do septo interventricular e parede livre de ventrículo esquerdo ( 1 caso); rotura da parede posterior do ventrículo esquerdo ( 1 caso); lesāo de aorta ascendente na abertura do tórax (esternotomia) ( 1 caso); dissecçāo aguda de aorta tipo I (1 caso); 5 casos de coagulopatia e sangramento incontrolável, e os pacientes fechados com compressas e 7 casos de baixo débito, sendo necessárias medidas de suporte como balāo intra-aórtico e/ ou bio-pump.

Hemorragia: Foi a principal causa de óbito em 16 pacientes. Sendo 13 reoperaçōes.

Em 13 casos foi realizada a troca de valva mitral isolada, sendo que em 4 era a quarta operaçāo, em 2 a terceira operaçāo, em 5 a segunda operaçāo e em 1 caso de primeira operaçāo. Dois pacientes com troca de valva aórtica, 1 a segunda operaçāo e 1 a primeira operaçāo. Um paciente com troca valvar mitral e aórtica era a segunda operaçāo e 1 caso com troca mitral e plástica de De Vega na tricúspide a terceira operação.

Dos pacientes com operaçōes prévias, em 9 casos o sangramento era difuso, nāo sendo encontrado o local de sangramento e dos 4 restantes, 1 caso de sangramento importante por laceração do átrio direito no local da bolsa da cava inferior; 1 caso de lesão da aorta na abertura do tórax por grande aderência aorto-esternal; 1 caso de rotura do sulco atrioventricular e finalmente hemorragia causada por rotura da parede posterior de ventrículo esquerdo acrescido da circulação extracorpórea prolongada.

Dos casos de reoperaçāo em que a hemorragia foi a principal causa de óbito em 5 pacientes foi necessário o fechamento do tórax com compressas, pois havia sangramento incontrolável.

Dos 3 pacientes que foram a óbito por sangramento e que não tinham operaçōes prévias, 1 caso tratava-se de dissecçāo aguda de aorta no local do jelco; neste caso houve circulaçāo extracorpórea prolongada seguida de choque hemorrágico por tentativa de reparo da lesão; 1 caso de laceração do septo interventricular e parede de ventrículo esquerdo, obrigando o retorno à circulaçăo extracorpórea (CEC) e evoluindo com coagulopatia importante; finalmente 1 caso de febre reumática ativa (cardite reumática) com aderências importantes e sangramento difuso.

Choque cardiogênico: Encontramos maior número de casos com choque cardiogênico (CC) nas causas clínicas (15 casos) do que nas causas anatomopatológicas (4 casos) de óbito.

Dos 15 casos o choque cardiogênico (10 mitrais, 5 aórticos), em 11 foi a causa direta do óbito, e em 4 contribuiu para o óbito.

De todos os pacientes com diagnóstico de CC, $70 \%$ evoluíram com refratariedade ao tratamento clínico ou mesmo com balāo intra-aórtico e/ou biopump.

Dos 10 pacientes mitrais, o estudo anatomopatológico mostrou em 1 caso obstrução da via de saída do ventrículo esquerdo pela prótese mitral mais infarto do miocárdio circunferencial em paciente de 4 anos de idade; 1 caso de dissecção aguda de aorta no ato operatório e que na correçāo o Teflon obstruiu o óstio da coronária direita, levando a infarto do miocárdio; 1 caso de troca de valva mitral mais revascularizaçäo do miocárdio (1 ponte de safena), evoluiu com baixo débito importante com a ponte de safena ocluída; 1 caso de troca da valva mitral por prótese mecânica que evoluiu com "travamento" e 6 casos de disfunçāo ventricular severa cujo estudo anatomopatológico mostrou infarto do miocárdio subendocárdicos em 3 deles.

Dos 5 pacientes aórticos, 1 paciente com endocardite em prótese levando a insuficiência aórtica apresentava comunicação interventricular; o segundo caso, submetido a ressecçāo de éstenose sub aórtica e plástica de valva aórtica, teve durante a operação rotura do septo interventricular e parede livre de ventrículo esquerdo; o terceiro caso de 
FABRI, H. A.; POMERANTZEFF, P. M. A.; MONTEIRO, A. C. M.; GUTIERREZ, P.; VIOLANTE, R.; AULER JÚNIOR, J. O. C.; GRINBERG, M.; VERGINELLI, G.; JATENE, A. D. - Análise do estudo anatomopatológico de pacientes submetidos a cirurgia valvar no InCór - HC FMUSP. Rev. Bras. Cir. Cardiovasc., 7(4):243-249, 1992.

troca de valva aórtica teve que voltar em CEC, para ampliaçāo do anel, a necropsia mostrou áreas de infarto do miocárdio subendocárdico. Constatou-se, ainda, 4 casos de infarto do miocárdio subendocárdico. Dois pacientes apresentaram arritmias severas com repercusāo hemodinâmica importante.

Infecçāo: Constatamos 10 casos de infecçāo, em 5 a causa principal do óbito, sendo 7 mitrais, 2 aórticos e 1 mitro-aórtico.

Com relaçāo aos mitrais, um paciente evoluiu em choque séptico com foco infeccioso no intracath; 1 paciente no $1^{\circ}$ dia de pós-operatório apresentou apendicite aguda abscedada; operado, evoluiu com septicemia por Staphylococcus aureus; 1 paciente com endocardite em prótese comprovada pelo ecocardiograma no $5^{\circ}$ dia de pós-operatório, foi reoperada para a retroca da prótese, evoluindo com mediastinite e óbito 7 dias após.

O restante dos mitrais apresentou infecção pulmonar comprovada por exame radiológico e necropsia. Nos aórticos, 1 caso de troca de valva aórtica por endocardite infecciosa na prótese, evoluiu com septicemia e embolia séptica por Staphylococcus epidermides, 1 caso de endocardite por fungos na prótese (Candida albicans), evoluindo com septicemia.

Um paciente com troca valvar mitral e aórtica por lesão reumática que evoluiu após a alta hospitalar ( $9^{9}$ pós-operatório) com quadro de afasia, hemiparesia completa à esquerda, embolia para membro superior direito, toxemia e febre, à necropsia revelou endocardite por germe gram positivo em prótese aórtica.

Complicaçōes pulmonares: Foram observadas em 7 casos, sendo 6 mitrais e 1 mitroaórtico.

Dentre os mitrais tivemos 3 casos de síndrome de angústia respiratória do adulto (S.A.R.A.), sendo que 1 deles foi constatado logo após a CEC; 2 casos de tromboembolismo pulmonar; 1 caso de hemotórax bilateral de grande volume (achado de necropsia), seguido de coagulopatia e sangramento. O paciente mitro-aórtico teve hemorragia alveolar maciça seguida, de septicemia por provável fragilidade capilar.

Falência de múltiplos órgảos: Foi a causa final de óbito em 6 pacientes, sendo 3 trocas mitrais isoladas, 2 pacientes mitro-aórticos e 1 mitrotricuspídeo.

É importante salientar que em todo os casos em que ocorreu falência de múltiplos órgãos ela foi seguida de sangramento importante e baixo débito prolongado.
Complicaçōes cerebrais: Ocorreram em 3 pacientes, sendo 1 troca mitral e 2 trocas aórticas. No paciente mitral a causa da morte foi acidente vascular cerebral hemorrágico (A.V.C.H.) e nos aórticos, 1 caso de hemorragia cerebral difusa maciça sugerindo aneurisma micótico roto e outro infarto cerebral hemorrágico sem causa aparente.

Arritmias: Arritmia ventricular severa foi considerada a causa primária de óbito em 2 pacientes. Um caso de troca de valva mitral evoluiu com taquiarritmia de difícil controle na sala de operaçōes e no pós-operatório imediato. A necropsia revelou infarto subendocárdico. No outro caso, uma troca mitral e aórtica à necropsia revelou fibrose do nó sinusal responsável pela severa arritmia.

Dissecçāo aguda da aorta: Num paciente submetido a troca da valva mitral isolada houve dissecçāo da aorta no local do jelco, indo a óbito na sala de operaçōes.

\section{COMENTÁRIOS}

Trabalhos analisando os resultados clínicos e hemodinâmicos no seguimento de pacientes submetidos a cirurgia valvar têm sido muito freqüentes, porém o estudo anatomopatológico dos pacientes que evoluíram para óbito hospitalar e a correlaçăo destes achados com a causa clínica do óbito não tem tido muitas vezes o destaque necessário.

Nossa casuística revelou que $63,63 \%$ dos pacientes tinham operaçōes valvares prévias e que $36,36 \%$ apresentaram distúrbios hemorrágicos graves, sendo que $11(25 \%)$ pacientes com operaçōes valvares prévias apresentavam alguma forma de hepatopatia.

A coagulaçāo é um dos pontos mais importantes do pós-operatório dos pacientes submetidos a algum tipo de cirurgia valvar. Freqüentemente apresentam disfunçāo hepática grave causada pela insuficiência cardíaca congestiva (I.C.C.), ou mesmo hepatite adquirida ao longo de operaçōes anteriores. A disfunção plaquetária aguda induzida pela CEC, a diluição dos fatores de coagulação e mesmo a perda temporária da funçāo hepática causada pela hipotermia determinam coagulopatias de difícil controle. A isto somam-se a politransfusão e a instabilidade hemodinâmica com todas as suas conseqüências.

ROBERTS \& MORROW ${ }^{15} \mathrm{em}$ estudo de 64 necropsias, mostraram que a hemorragia foi a segunda maior causa de óbito no pós-operatório, seguida da disfunçāo de prótese valvar.

Sempre que possível a compensaçāo do paciente é feita no pré-operatório, porém devido ao grau 
FABRI, H. A.; POMERANTZEFF, P. M. A.; MONTEIRO, A. C. M.; GUTIERREZ, P.; VIOLANTE, R.; AULER JÚNIOR, J. O. C.; GRINBERG, M.; VERGINELLI, G.; JATENE, A. D. - Análise do estudo anatomopatológico de pacientes submetidos a cirurgia valvar no InCór - HC FMUSP. Rev. Bras. Cir. Cardiovasc., 7(4):243-249, 1992.

de insuficiência cardíaca causada pela própria valvopatia, nem sempre o estado ideal é conseguido.

Acreditamos ser importante a busca de novos métodos para melhor avaliação no pré-operatório, pois sabemos que o trinômio: reoperaçāo-coagulopatia-hepatopatia é o responsável por grande número de óbitos.

Outra grande causa de óbito no pós-operatório precoce na nossa casuística foi o choque cardiogênico.

SCHOEN et alii ${ }^{21}$ descreveram 262 casos de óbito no pós-operatório precoce e que as causas mais comuns foram falência cardíaca na sala de operaçōes, baixo débito e arritmias.

HUSEBYE et alii ${ }^{4}$ analisando fatores de risco em 552 reoperaçōes de próteses valvares mostraram em pacientes em classe funcional IV (NYHA) a mortalidade de $21 \%$ para a valva aórtica e $41 \%$ para a valva mitral; pacientes operados em caráter de emergência em classe funcional IV a mortalidade para os aórticos foi de $56 \%$ e para os mitrais foi de $67 \%$.

$\mathrm{Na}$ nossa casuística encontramos $87 \%$ dos pacientes em classe funcional III e IV mostrando que a mortalidade está ligada diretamente ao estado clínico do paciente.

Como vimos, 4 casos de infarto agudo do miocárdio nāo foram comprovados clinicamente, sendo que 3 contribuíram diretamente para o óbito através de choque cardiogênico.

O choque cardiogênico que se traduz em baixo débito algumas vezes é causado por infarto subendocárdico não diagnosticado no pós-operatório e provavelmente reflete uma proteçāo miocárdica deficiente.

LAURINDO et alii ${ }^{8}$ estudando 662 necropsias depois de troca valvar, constataram 12 infartos de miocárdio, 6 sem causa definida; em 4 deles havia necrose maciça circunferencial, principalmente subendocárdica.

As infecçōes sāo causas importantes de óbito no pós-operatório de cirurgia valvar estando associadas a pácientes com alto potencial em desenvolvêlas, já que além dos fatores predisponentes gerais sāo submetidos a procedimentos invasivos, tais como entubação, anestesia geral e o próprio ato operatório.

ZEIEN \& KLATT ${ }^{25}$ estudando 96 necropsias de cirurgia valvar mostraram óbito por infecçāo em $22 \%$ dos casos.

ROBERTS \& HAMMER ${ }^{14}$ relataram que em 46 estudos anatomopatológicos de pacientes submetidos a cirurgia valvar 3 óbitos deveram-se a infecçăo.

Em nossa casuística $11 \%$ dos óbitos constatados no estudo anatomopatológico foram por infecçāo, chamando a atençāo para o cuidado especial que se deve ter neste tipo de paciente.

Vários autores 2, 15, 21, 24 citam como causa de óbito no pós-operatório de cirurgia valvar a arritmía, embora sempre associada a drogas, hipoxia, infarto do miocárdio, má proteçāo do miocárdio. Na nossa casuística em 2 pacientes foi responsável direta pelo óbito, em 1 sem causa aparente e em outro a necropsia revelou fibrose do nó sinusal.

Podemos concluir da nossa análise que houve correlação entre o diagnóstico clínico e anatomopatológico dos óbitos em 36 (82\%) pacientes.

Nos pacientes com choque cardiogênico em que nāo houve correlaçāo clínico-patológica, o infarto subendocárdico teve papel fundamental.

É importante salientar o grande número de pacientes que apresentava hepatopatia crônica. 
FABRI, H. A.; POMERANTZEFF, P. M. A.; MONTEIRO, A. C. M.; GUTIERREZ, P.; VIOLANTE, R.; AULER JÚNIOR, J. O. C.; GRINBERG, M.; VERGINELLI, G.; JATENE, A. D. - Análise do estudo anatomopatológico de pacientes submetidos a cirurgia valvar no InCór - HC FMUSP. Rev. Bras. Cir. Cardiovasc., 7(4):243-249, 1992.

\section{RBCCV 44205-185}

FABRI, H. A.; POMERANTZEFF, P. M. A.; MONTEIRO, A. C. M.; GUTIERREZ, P.; VIOLANTE, R.; AULER JR., J. O. C.; GRINBERG, M.; VERGINELLI, G.; JATENE, A. D. - Anatomopathological study of patients submitted to valvar surgery at InCór-HC FMUSP. Rev. Bras. Cir. Cardiovasc., 7(4):243-249, 1992.

ABSTRACT: Of the 44 necropsies which were carried out in patients submitted to valvar surgery in 1990 at InCór (Instituto do Coraçāo), the following clinical causes of death were found in Mitral patients: coagulopathy and bleeding in 11 cases; low cardiac output in 7; cardiogenic shock in 5; septic shock in 3; failure of multiple organs in 3; acute respiratory distress syndrom (ARDS) in 2; severe ventricular arrythmia in 2, and pulmonary thromboembolism in 1 . In aortic patients: coagulopathy and bleeding in 5 cases; cardiogenic shock in 2; septicaemia in 3; ruptured micotic aneurism in 1; stroke in 1, and ventricular arrhythmia in 1 . In mitroaortic patients: coagulopathy and bleeding in 2 cases; pulmonary thromboembolism in 2; septicaemia in 1; severe ventricular arrythmia in 1, and ARDS in 1. The following anatomopathological causes of death were observed. In mitral patients: hemorrhagic shock in 13 cases; failure of multiple organs in 4; septic shock in 3; broncopneumonia in 4; acute myocardial infarction (AMI) in 3; cardiogenic shock in 3; cerebral hemorrahage in 1, and ARDS in 1. In aortic patients: endocarditis in 3 cases: hemorrhagic shock in 2; cerebral hemorrhage in 2; AMI in 1; cardiogenic shock in 1; septicaemia in 1; acute respiratory distress syndrom (ARDS) in 1, and acute dissection of the aorta, with coagulopathy in 1 . In mitroaortic patients: failure of multiple organs in 2 cases; pulmonary thromboembolism in 2; ARDS in 1; hemorrhagic shock in 1, and endocarditis in 1. We concluded that there was correlation between the clinical and anatomopathological diagnoses in 36 patients, corresponding to $82 \%$ of the cases, the principal cause of death being due to hemorrhagic shock. An important observation which was made in this study, was that $11(25 \%)$ patients had some form of chronic hepatopathy which had not previously diagnosed from the clinical laboratory study. Some of the patients which developed clinically unexplained low cardiac output presented subendocardial infarct as an anatomopathological finding.

DESCRIPTORS: heart valves, anatomopathological study; heart valves, surgery.

\section{REFERÊNCIAS BIBLIOGRÁFICAS}

1 BERGGREN, H. R. E.; HERLITZ, J.; HJALMARSON, A.; WALDENSTROM, A.; WALDENSTROM, J.; OLSSON, G. W. - Improved myocardial protection during cold cardioplegia by means of increased myocardial glycogen stores. Thorac. Cardiovasc. Surgeon, 30: 389-392, 1982.

COLAPINTO, N. D. \& SILVER, M. D. - Prosthetic heart valve replacement: causes of early postoperative death. J. Thorac. Cardiovasc. Surgeon, 61: 938944, 1971.

GONZALEZ - LAVIN, L.; GONZALEZ - LAVIN, J.; McGRATH, L. B.; AMINI, S.; GRAF, D. - Factors determining in-hospital or late survival after aortic valve replacement. Chest, 95: 38-42, 1989.

HUSEBYE, D. G.; PLUTH J. R., PIEHLER, J. M.; HARTZELL, V. S.; ORSZULAK, T. A.; PUGA, F. J.; DANIELSON, G. K. - Reoperation on prosthetic heart valves: an analysis of risk factors in $\mathbf{5 5 2}$ patients. J. Thorac. Cardiovasc. Surg., 86: 543-552, 1983.

KIRKLIN, J. W.; CONTI, V. R.; BLACKSTONE, E. H. Prevention of myocardial damage during cardiac operations. N. Engl. J. Med., 301: 135-141, 1979.
KIRKLIN, J. W.; BROWN, R. C.: Risk factors for mortality after primary combined valvular and coronary artery surgery. Circulation, 79 (Supl. 1) I$185-1-190,1989$

KYOSOLA, K. T.; BRAIMBRIDGE, M. W.; DANACOTT - CANKOVIC, S.; CHAMBERS, D. J. - Myocardial catecholamines following cold cardioplegic arrest during open - heart surgery. Scand. J. Thor. Cardiovasc., 18: 209-215, 1984.

8 LAURINDO, F. R. M.; GRINBERG, M.; ASSIS, R. V. C. JATENE, A. D.; PILEGGI, F. - Perioperative acute myocardial infarction after valve replacement. $A m$. J. Cardiol., 59: 639-642, 1987.

LUND, O. - Preoperative risk evaluation and stratification of long-term survival after valve replacement for aortic stenosis; reasons for earlier operative intervention. Circulation, 82: 124-139, 1990.

McGREGOR, C. G. A.; MAC LEOD, M. D.; MUIR, A. L.; SAITH, A. F.; HANNAN, W. J.; MILLER, H. C. Myocardial infarction related to valve replacement surgery. Br. Heart J., 51: 612-617, 1984.

11 MORALES, A. R.; FINE, G.; TABER, R. E. - Cardiac surgery and myocardial necrosis. Arch. Path., 83: 71-79, 1967. 
FABRI, H. A.; POMERANTZEFF, P. M. A.; MONTEIRO, A. C. M.; GUTIERREZ, P.; VIOLANTE, R.; AULER JÚNIOR, J. O. C.; GRINBERG, M.; VERGINELLI, G.; JATENE, A. D. - Análise do estudo anatomopatológico de pacientes submetidos a cirurgia valvar no InCór - HC FMUSP. Rev. Bras. Cir. Cardiovasc., 7(4):243-249, 1992.

POMERANTZEFF, P. M. A.; ABREU, M. C. S.; AMATO, M.; MORETTI, M.; AULER JR., J. O. C.; GRINBERG, M.; TARASOUTCHI, F.; MANSUR, A.; DIAS, A. R.; BITTENCOURT, D.; STOLF, N.; VERGINELLI, G.; JATENE, A. D. - Retroca valvular. Rev. Bras. Cir. Cardiovasc., 2: 180-188, 1987.

POMERANTZEFF, P. M. A.; KIOKA, Y.; KAWABE, L. T.; AULER JR, J. O. C.; LOPES, E. A.; VERGINELLI, G.; JATENE, A. D. - Obstruçāo da via de saída de ventrículo esquerdo por prótese mitral: relato de seis casos. Rev. Bras. Cir. Cardiovasc., 5: 120-124, 1990.

ROBERTS, W. C. \& HAMMER, W. J. - Cardiac pathologic after valve replacement with a tilting disc prosthesis. (Björk - Shilley type): a study of 46 necropsy patients and 49 (Björk - Shilley prosthesis. Am. J., Cardiol., 37: 1024-1033, 1976.

ROBERTS, W. C. \& MORROW, A. G. - Causes of early postoperative death following cardiac valve replacement, clinic - pathologic correlations in 64 patients studied at necropsy. J. Thoracic. Cardiovasc., Surg., 54: 422-437, 1967.

ROBERTS, W. C. \& MORROW, A. G. - Late postoperative pathological findings after cardiac valve replacement. Circulation, 35 e 36, (Supl. 1) |-48-I-62, 1967.

ROBERTS, W. C. \& SULLIVAN, M. F. - Clinical and necropsy observations early after simultaneous replacement of the mitral and aortic valves. Am. J. Cardiol., 58: 1067-1084, 1986.

ROBERTS, W. C.\& SULLIVAN, M. F. - Combined mitral valve stenosis and tricuspid valve stenosis: morphologic observations after mitral and tricuspid valve

replacement or mitral replacement and tricuspid valve commissurotomy. Am. J. Cardiol., 58: 850-852, 1986.

19

ROSE, A. G. \& PATH, M. R. C.. - Etiology of acquired valvular heart disease in adults; a survey of 18,132 autopsies and 100 consecutive valve replacement operations. Arch. Pathol. Lab. Med., 110: 385-388, 1986.

SALOMON, N. W.; STINSON, E. B.; GRIEPP, R. B.; SHUNWAY, N. E. - Mitral valve replacement: longterm evaluation of prosthesis related mortality and morbidity. Circulation, 56 (Supl.3) II-94 II-101, 1977.

SCHOEN, F. J.; TITUS, J. L.; LAWRIE, G. M. - Autopsy - determined causew of death after cardiac valve replacement. JAMA, 249: 899-902, 1983.

SCHWARZ, F.; FLAMENG, W.; SCHARPER, J.; LANGEBARTELS, F.; SESTO, M.; HEHRLEIN, F.; SCHLEPPER, M. - Miocardial structure and function in patients with aortic valve disease and their relation to postoperative results. Am. J. Cardiol., 41: 661. $669,1978$.

SULLIVAN, M. F. \& ROBERTS, W. C. - Clinical and morphologic observations after simultaneous replacement of the tricuspid, mitral and aortic valves. Am. J. Cardiol., 58: 781-789, 1986.

24 TEASDALE, S. J.; ZULYS, V. J.; MYCYK, T.; BAIRD, R. J.; GLYNN, M. F. - Ancrod anticoagulation for cardiopulmonary bypass in heparin - induced thrombocytopenia and thrombosis. Ann. Thorac. Surg., 48: $712-713,1989$.

ZEIEN, L. B. \& KLATT, E. C. - Cardiac valve prosthesis at autopsy. Arch. Pathol. Lab. Med., 114: 933-937, 1990. 